\title{
INFESTAÇÃO DE BEMISIA TABACI (GENN.) BIÓTIPO \\ B (HEMIPTERA: ALEYRODIDAE) E CALIOTHRIPS PHASEOLI \\ (HOOD.) (THYSANOPTERA: THRIPIDAE) EM GENÓTIPOS DE FEIJOEIRO
}

\section{F.G. Jesus ${ }^{1 *}$, A.L. Boiça Junior ${ }^{1}$, S.A.M. Carbonel ${ }^{2}$, C.P. Stein ${ }^{3}$, R.M. Pitta ${ }^{1}$}

${ }^{1}$ Universidade Estadual Paulista, Faculdade deCiências Agráriase Veterinárias, Departamento de Fitossanidade, Via de Acesso Prof. Paulo Donato Castellane s/no, CEP 14884-900, Jaboticabal, SP, Brasil. E-mail fgj@cefeturutai.edu.br

\section{RESUMO}

Este trabalho teve por objetivo avaliar a infestação de Bemisia tabaci (Genn) biótipo B e de Caliothrips phaseoli (Hood.) em quinze genótipos de feijoeiro em condições de campo. Os genótipos utilizados foram: IAPAR 31, Rosinha G2, Jalo precoce, Pérola, IAC Harmonia, Gen 99TGR110, Gen 99TG2868, Gen 99TGR3416, Gen 99TG3450, Gen 99TG823, Gen 99TGR609, IAC Jaraguá, Gen 95A10061531, Gen 99TGR3114 e Gen 96A1473153V2. O experimento foi conduzido nos meses de maio a julho de 2006, utilizando delineamento de blocos casualizados com 15 tratamentos e quatro repetições. Foram realizadas avaliações semanais totalizando quatro amostragens, contando-se o número de ovos e ninfas de B. tabaci biótipo B e ninfas de C. phaseli em dez folíolos. Dentre os genótipos estudados, os menos ovipositados por B. tabaci biótipo B foram IAC Harmonia, Pérola, Gen TG3114 e Gen 95A10061531, enquanto os mais ovipositados foram IAC Jaraguá e Gen 99TG3450. As menores infestações de ninfas de B. tabaci biótipo B foram observadas em Pérola e IAC Harmonia e maior em IAC Jaraguá. Não foi verificada correlação linear significativa entre temperatura média semanal e número total de ninfas de B. tabacibiótipo B e C. phaseoli.

PALAVRAS-CHAVE: Phaseolus vulgaris, mosca-branca, resistência de plantas, tripes.

\section{ABSTRACT}

INFESTATION OF BEMISIA TABACI (GENN.) BIOTYPE B (HEMIPTERA: ALEYRODIDAE) AND CALIOTHRIPS PHASEOLI (HOOD.) (THYSANOPTERA: THRIPIDAE) IN BEANS GENOTYPES. The objective of this work was to evaluated the infestion of the whitefly Bemisiatabaci (Genn.) B biotype and thrips Caliothrips phaseoli (Hood) in fifteen beans genotypes in field condition. The genotypes IAPAR 31, Rosinha G2, Jalo precoce, Pérola, IAC Harmonia, Gen 99TGR110, Gen 99TG2868, Gen 99TGR3416, Gen 99TG3450, Gen 99TG823, Gen 99TGR609, IAC Jaragua, Gen 95A10061531, Gen 99TGR3114 e Gen 96A1473153V2 was used. The experiment was conducted from May to July 2006. The statistical design was randomized blocks, totalizing 15 treatments and four replications. Evaluation was realized weekly, totalizing seven samplings. The evaluations were accomplished on a weekly basis by counting $B$. tabaci biotype B eggs and nymphs and nymphs of C. phaseoli in 10 leaflets per plot. The less oviposition genotypes by $B$. tabaci biotype B were IAC Harmonia, Pérola, Gen TG3114 e Gen 95A10061531, while the most oviposited were IAC Jaraguá and Gen 99TG3450. The less presence of nymphs of whitefly were observed on Pérola and IAC Harmonia and the most at IAC Jaraguá. It was observed a negative and non significant linear correlation between average temperature versus number of silverleaf whitefly and temperature versus number of thrips was verified.

KEY WORDS: Phaseolus vulgaris, silverleaf whitefly, host plant resistance, thrips.

\footnotetext{
${ }^{2}$ Instituto Agronômico, Centro de Pesquisa e Desenvolvimento de Grãos e Fibras, Campinas, SP, Brasil. ${ }^{3}$ Instituto Agronômico, Centro de Pesquisa e Desenvolvimento de Fitossanidade, Campinas, SP, Brasil. *Bolsista CAPES.
} 


\section{INTRODUÇÃO}

O feijoeiro pode sofrer o ataque de insetos eácaros que afetam a produção antes e após a colheita, sendo que as perdas causadas variam de 33 a $86 \%$ na produção final (YОКОYAMA, 2006).

Oataque deinsetosé prejudicial desde a semeadura do feijoeiro, passando pelas fases vegetativas e reprodutivas das plantas, e até após a colheita podem ocorrer danos aos produtos armazenados (MAGALhães; CARValho, 1998). Dentre essas pragas destacam-se a mosca-branca Bemisia tabaci (Gennadius 1889) biótipo B (Hemiptera: Aleyrodidae) e o tripes Caliothrips phaseoli (Hood 1912) (Thysanoptera: Trhipidae) que atacam as folhas das plantas.

A mosca-branca B. tabaci biótipo B causa sérios prejuízos em muitas áreas produtoras do Brasil, através da transmissão do vírus do mosaico dourado (VMDF) e outros vírus (SALGUERO, 1993), doenças essas que limitam a produção do feijão, podendo causar perdas de até100\% (FARIA;ZIMMERMANN, 1987; FARIAet al., 1994; CZEPAK et al., 1999), além disso, provocam danos diretos pela sucção e extração de seiva elaborada (GALLO et al., 2002)

Entre as causas da alta incidência da moscabranca estão a expansão da área de plantio da soja, uma das hospedeiras preferenciais do inseto, a ampliação da época de semeadura e os cultivos sucessivos e escalonados do feijoeiro com o uso de pivô-central (VIEIRA et al., 1998).

A seleção de plantas resistentes a mosca-branca representa uma forma importante de controle visando reduzir as perdas causadas por esses insetos (McAuslane, 1996).

Além da mosca-branca, os tripes também são importantes pragas do feijoeiro. A espécie C. phaseoli é normalmente encontrada em culturas de feijão e ervilha e seus danos diretos são decorrentes da sucção de seiva sendo que em ataques intensos as folhas tornam-se deformadas, amareladas, secamecaem (GALLO et al., 2002).

O controle das pragas do feijoeiro tem sido feito quase que exclusivamente por inseticidas e por controle cultural. PRABHAKER et al. (1985) mencionaram, entretanto, que características biológicas e comportamentais dos insetos, como rápido desenvolvimento, alta fecundidade e grande capacidade de dispersão, são fatores que aumentam a probabilidade de aparecimento de resistência aos inseticidas comerciais de diferentes grupos químicos (PrABHAKER et al., 1989; DitTrich et al., 1990). Em razão desse e de outros problemas causados pelos inseticidas no agroecossistema, métodos alternativos de controle de pragas vêm sendo estudados.

BOIÇA JÚNIOR; VENDRAMIM (1986) observaram que a cultivar Bolinha modificou o ciclo de vida deB.tabaci, sugerindo a existência de resistência do tipo antibiose. Nas cultivares Carioca e G-2618 o desenvolvimento foi favorecido; na linhagem BAT 85 e na variedade Goiano Precoce ocorreu maior oviposição; e na linhagem BAT 63 houve reduzido número de ninfas e menor preferência para oviposição. FARIA et al. (1998) ressaltram que trabalhos desenvolvidos em Goiás levaram à recomendação da cultivar Ônix para cultivo naépoca da seca. No Instituto Agronômico do Paraná (Iapar) foram desenvolvidas algumas cultivares moderadamente resistente ao vírus do mosaico dourado, como a IAPAR 57 e a IAPAR MD 820. LEMOs et al. (2003) verificaram que os genótipos Ônix, IAPAR 57, IAPAR 65 e IAPAR 72 foram poucos infestados pela mosca-branca em condições de campo.

Assim o presente trabalho teve como objetivo estudar a infestação de B. tabaci biótipo B e C. phaseoli em diferentes genótipos de feijoeiro, procurando encontrar possíveis fontes de resistência as esses insetos.

\section{MATERIAL E MÉTODOS}

O experimento foi instalado e conduzido no período de maio a julho de 2006 em área experimental do Departamento de Fitossanidade da Faculdade de Ciências Agrárias e Veterinárias da Universidade Estadual Paulista (UNESP), Jaboticabal, SP.

O espaçamento de plantio da cultura foi de $0,50 \mathrm{~m}$ na entre linha, com densidade aproximada de 12 plantas por metro linear. Na adubação de plantio foram utilizados $430 \mathrm{~kg}$.ha- ${ }^{1}$ da fórmula 04-14-08, em cobertura 180 kg.ha- $^{-1}$ de sulfato de amônia dividido em duas etapas, a primeira com 15 dias e a segunda ao redor dos 30 após a emergência.

O delineamento experimental adotado foi de blocos casualizados com quinze tratamentos correspondentes aos genótipos IAPAR 31, Rosinha G2, Jalo precoce, Pérola, IAC Harmonia, Gen 99TGR110, Gen 99TG2868, Gen 99TGR3416, Gen 99TG3450, Gen 99TG823, Gen 99TGR609, IAC Jaraguá, Gen 95A10061531, Gen 99TGR3114 eGen 96A1473153V2 em quatro repetições.

Cada parcela foiconstituída de quatrolinhas de $4 \mathrm{~m}$ de comprimento, com $8 \mathrm{~m}^{2}$ de área total $4 \mathrm{~m}^{2}$ de área útil, tendo-se como área total do experimento $384 \mathrm{~m}^{2}$.

As amostragens de incidência de mosca-branca e tripes foram realizadas semanalmente, (4 avaliações) após a emergência das plantas (DAE), coletando-se dez folíolos da parte mediana da planta por parcela e acondicionando-os em sacos de papel com capacidade para dois litros. Em seguida, as amostras foram encaminhadas para laboratório e, com auxílio de um microscópio estereoscópico, foram quantificados o número de ovos e de ninfas de $B$. tabaci biótipo $B$ e de ninfas de C. phaseoli. 
Os dados referentes à contagem do número de ovos e ninfas de $B$. tabaci biótipo B e das ninfas de $C$. phaseoli em plantas de feijoeiro foram submetidos à análise de variância pelo teste $\mathrm{F}$, e as médias separadas pelo teste de Tukey. Para analise, os dados originais foram transformados em $(x+0,50)^{1 / 2}$.

A variável climática temperatura média semanal foi correlacionada com o número total de ninfas de mosca-branca e número total de ninfas de tripes, através de uma análise estatística do tipo regressão linear. Nãoforam considerados os dados referentes à precipitação pluviométrica devido aos baixos valores.

\section{RESULTADOS E DISCUSSÃO}

Verificaram-se diferenças significativas aos 21, 28, 35 e 42 dias após a emergência das plantas (DAE) para o número médio de ovos de $B$. tabaci biótipo $B$ (Tabela 1).

Na avaliação realizada aos 21 DAE, destacou-se o genótipo IAC Jaraguá como o mais ovipositado, com média de 62,2 ovos em dez folíolos amostrados, e com menor preferência para oviposição destacaram-se os genótipos IAC Harmonia, Gen 99TGR110, Gen TGR3114 e Pérola, com médias de 17,5; 21,7; 23,7 e 24, respectivamente.

Aos 28DAE, observa-sequeogenótipoIAC Jaraguá continua sendo o mais preferido pela mosca-branca para oviposição, com 74,0 ovos em média, e os genótipos Gen 95A10061531, Gen TGR3114, Rosinha G2, Gen 99A1473153V2, Gen 99TG883, IAC Harmonia, Pérola e Gen 99TG2868 os menos preferidos para oviposição pela praga com 5,$5 ; 5,5 ; 5,7 ; 6,0 ; 6,2 ; 6,5 ; 7,5$ e 7,5 ovos, respectivamente.

Para a avaliação aos 35 DAE, observa-se que ocorreu diferença significativa entre os genótipos estudados, sendo a maior oviposição no genótipo Gen 96A1473153V2, com 31,2 ovos em média, enquanto que os genótipos IAC Harmonia, Gen 95A10061531, Gen TGR3114, Pérola e Rosinha G2 comportaram-se de forma contrária, apresentando menores oviposições com 0,$7 ; 1,5 ; 4,2 ; 5,2$; e 5,7 ovos em média, respectivamente, nos dez folíolos avaliados.

BOIÇA JúNIOR et al. (2000), observando a interação de variedades de feijoeiro com adubação e uso de inseticidas no controle da mosca-branca na safra "de inverno", encontraram baixas infestações de ovos e ninfas dessa praga, constatando maiores infestações entre 14 e 28 dias após a emergência das plantas.

Aos 42 DAE, destacou-se o genótipo Gen 99TG3450 como o mais suscetível, com 19,0 ovos em média, enquanto que os genótipos menos preferidos para oviposição foram Gen 95A10061531, Pérola e Gen99TGR609 com 1,2;1,7e2,0 ovos, respectivamente, nos dez folíolos analisados.

Tabela 1 - Média do número de ovos de Bemisia tabaci (Genn.) biótipo B em dez folíolos, obtidos em 15 genótipos de feijoeiro, em quatro amostragens, Jaboticabal, SP, 2006.

\begin{tabular}{|c|c|c|c|c|}
\hline \multirow[t]{2}{*}{ Genótipo } & \multicolumn{4}{|c|}{ Dias após a emergência das plantas ${ }^{1,2}$} \\
\hline & 21 dias & 28 dias & 35 dias & 42 dias \\
\hline 1- IAPAR 31 & $40,0 \pm 7,4 \mathrm{ab}$ & $60,5 \pm 16,9 a b$ & $15,2 \pm 6,4 \mathrm{ab}$ & $10,7 \pm 4,3 \mathrm{ab}$ \\
\hline 2- Rosinha G2 & $29,5 \pm 5,7 \mathrm{ab}$ & $5,7 \pm 2,5 \mathrm{~d}$ & $5,7 \pm 1,2 \mathrm{~d}$ & $4,7 \pm 1,3 \mathrm{ab}$ \\
\hline 3- Jalo precoce & $30,7 \pm 5,7 \mathrm{ab}$ & $21,7 \pm 6,2 \mathrm{bcd}$ & $8,7 \pm 4,3 \mathrm{bc}$ & $6,0 \pm 1,7 \mathrm{ab}$ \\
\hline 4- Pérola & $24,0 \pm 3,5 \mathrm{~b}$ & $7,5 \pm 3,4 \mathrm{~d}$ & $5,2 \pm 1,8 \mathrm{~d}$ & $1,7 \pm 0,5 \mathrm{~b}$ \\
\hline 5- IAC Harmonia & $17,5 \pm 2,7 b$ & $6,5 \pm 1,5 \mathrm{~d}$ & $0,7 \pm 0,5 \mathrm{~d}$ & $3,5 \pm 1,8 \mathrm{ab}$ \\
\hline 6- Gen 99TGR110 & $21,7 \pm 2,5 b$ & $14,5 \pm 4,9 \mathrm{~cd}$ & $7,2 \pm 2,5 \mathrm{bc}$ & $7,5 \pm 2,5 \mathrm{ab}$ \\
\hline 7- Gen 99TG2868 & $30,7 \pm 3,8 \mathrm{ab}$ & $7,5 \pm 2,5 \mathrm{~d}$ & $10,7 \pm 2,8 \mathrm{bc}$ & $8,2 \pm 3,8 \mathrm{ab}$ \\
\hline 8- Gen 99TGR3416 & $39,7 \pm 5,4 \mathrm{ab}$ & $17,2 \pm 6,6 \mathrm{bcd}$ & $15,5 \pm 4,3 \mathrm{ab}$ & $9,7 \pm 4,0 \mathrm{ab}$ \\
\hline 9- Gen 99TG3450 & $49,2 \pm 11,9 a b$ & $59,7 \pm 20,9 a b c$ & $23,0 \pm 5,3 \mathrm{ab}$ & $19,0 \pm 4,1 \mathrm{a}$ \\
\hline 10- Gen 99TG883 & $41,0 \pm 10,0 \mathrm{ab}$ & $6,2 \pm 1,4 \mathrm{~d}$ & $9,0 \pm 1,4 \mathrm{bc}$ & $3,0 \pm 1,5 \mathrm{ab}$ \\
\hline 11- Gen 99TGR609 & $39,0 \pm 10,7 \mathrm{ab}$ & $16,0 \pm 4,3 \mathrm{~cd}$ & $9,0 \pm 1,3 \mathrm{bc}$ & $2,0 \pm 1,1 \mathrm{~b}$ \\
\hline 12- IAC Jaraguá & $62,2 \pm 7,3 \mathrm{a}$ & $74,0 \pm 9,5 \mathrm{a}$ & $26,0 \pm 15,6 a b$ & $16,7 \pm 13,4 \mathrm{ab}$ \\
\hline 13- Gen 95A10061531 & $27,7 \pm 5,9 \mathrm{ab}$ & $5,5 \pm 2,2 \mathrm{~d}$ & $1,5 \pm 0,5 \mathrm{~d}$ & $1,2 \pm 0,6 \mathrm{~b}$ \\
\hline 14- Gen TGR3114 & $23,7 \pm 5,9 b$ & $5,5 \pm 1,3 \mathrm{~d}$ & $4,2 \pm 1,4 \mathrm{~d}$ & $4,0 \pm 1,1 \mathrm{ab}$ \\
\hline 15- Gen 96A1473153V2 & $26,0 \pm 6,2 \mathrm{ab}$ & $6,0 \pm 2,1 \mathrm{~d}$ & $31,2 \pm 22,6 \mathrm{a}$ & $4,7 \pm 2,5 \mathrm{ab}$ \\
\hline F (Tratamentos) & $2,87^{* *}$ & $9,09^{* *}$ & $2,28^{*}$ & $2,35^{*}$ \\
\hline C. V. & 19,97 & 35,54 & 48,44 & 45,61 \\
\hline
\end{tabular}

1- Médias seguidas da mesma letra na coluna não diferem entre si pelo teste de Tukey a 5\% de probabilidade. 2- Para análise os dados foram transformados em $(x+0,50)^{1 / 2}$. 
ToscANo et al. (2002), estudaram a oviposição de adultos de $B$. tabaci biótipo B em tomateiro e CAMPOS et al. (2005) em algodoeiro, observaram que essa praga tem preferência por oviposição em plantas com 30 dias após a emergência, preferindo folhas mais novas. JEsus et al. (2006), avaliando a nãopreferência para oviposição da mosca-branca em dezenove genótipos de feijoeiro em condições de campo, também observaram que é nesta fase de desenvolvimento das plantas (ao redor de 30 dias) que a praga realiza maiores oviposições. Observando os diferentes autores com suas respectivas pesquisas, podemos destacar também a maior preferência da praga em ovipositar na planta nesta idade no presente trabalho.

Essa maior oviposição nesta época provavelmente ocorre devido o inseto encontrar constituição química e/ou morfológica mais favorável em razão da idade da planta (WAlker; Perring, 1994), além dos estímulos envolvidos entre o inseto e a planta (LARA, 1991).

Para os dados referentes a ninfas de mosca-branca, verificam-se diferenças entre os genótipos aos 28 DAE e 42 DAE (Tabela 2).

$\mathrm{Na}$ a avaliação realizada aos $21 \mathrm{DAE}$, observa-se que os genótipos estudados não diferiram entre si, mas destacam-se os genótipos IAPAR31, Jalo Precose,
Gen 99TGR3416, Gen 99TG3450, Gen 99TGR609 e Gen 96A1473153V2 que não apresentaram infestação da mosca-branca. Já o genótipo Gen TGR3114 mostrou tendência de maior preferência para infestação da praga com 15,2 ninfas em média.

$\mathrm{Na}$ avaliação do número de ninfas aos $28 \mathrm{DAE}$, visualiza-se diferença entre os tratamentos, sendo que o genótipo IAC Jaraguá comportou-se como o mais suscetível a infestação da praga, com 68,2 ninfas em média, os genótipos Gen 95A10061531, Gen 96A1473153V2, Pérola, Gen TGR3114 e Jalo Precoce comportaram-se como os menos preferidos, com 5,0; 5,2; 7,2; 8,5 e 9,2 ninfas em média, respectivamente, nos dez folíolos avaliados.

Aos 35 DAE não houve diferença entre os tratamentos, mas pode-se, contudo, destacar a tendência de maiores infestações nos genótipos IAPAR31 eGen 96A1473153V2, ambos com 20,7 ninfas de moscabranca em média, e os genótipos IAC Harmonia eGen TGR3114 com tendências de menores infestações com 2,2 e 4,2 ninfas de mosca-branca em média, respectivamente.

Em relação aos dados de ninfa aos 42 DAE, o genótipo IAC Jaraguá manteve-se como o mais suscetível à infestação da mosca-branca, com 17,2 ninfas em média, e o genótipo IACHarmonia o mais resistente, com 0,7 ninfas em média.

Tabela 2 - Média do número de ninfas de Bemisia tabaci (Genn.) biótipo B em dez folíolos, obtidos em 15 genótipos de feijoeiro, em quatro amostragens, Jaboticabal, SP, 2006.

\begin{tabular}{|c|c|c|c|c|}
\hline \multirow[t]{2}{*}{ Genótipo } & \multicolumn{4}{|c|}{ Dias após a emergência das plantas ${ }^{1,2}$} \\
\hline & 21 dias & 28 dias & 35 dias & 42 dias \\
\hline 1- IAPAR 31 & $0,00 \pm 0,0$ & $34,7 \pm 8,5 \mathrm{abc}$ & $20,7 \pm 12,6$ & $13,2 \pm 9,0 \mathrm{ab}$ \\
\hline 2- Rosinha G2 & $1,00 \pm 1,0$ & $14,0 \pm 1,4 \mathrm{bc}$ & $5,2 \pm 5,0$ & $2,5 \pm 0,6 \mathrm{ab}$ \\
\hline 3- Jalo precoce & $0,00 \pm 0,0$ & $9,2 \pm 3,3 c$ & $6,2 \pm 3,6$ & $4,0 \pm 1,4 \mathrm{ab}$ \\
\hline 4- Pérola & $0,25 \pm 0,3$ & $7,2 \pm 3,3 c$ & $6,0 \pm 1,9$ & $3,2 \pm 1,3 \mathrm{ab}$ \\
\hline 5- IAC Harmonia & $1,75 \pm 1,1$ & $13,7 \pm 1,6 \mathrm{bc}$ & $2,2 \pm 2,4$ & $0,7 \pm 0,5 b$ \\
\hline 6- Gen 99TGR110 & $0,75 \pm 0,8$ & $29,7 \pm 2,3 a b c$ & $8,5 \pm 6,1$ & $8,5 \pm 4,3 \mathrm{ab}$ \\
\hline 7- Gen 99TG2868 & $0,50 \pm 0,5$ & $23,2 \pm 1,5 \mathrm{abc}$ & $6,7 \pm 5,4$ & $8,5 \pm 1,4 \mathrm{ab}$ \\
\hline 8- Gen 99TGR3416 & $0,00 \pm 0,0$ & $31,2 \pm 6,5 a b c$ & $18,0 \pm 19,6$ & $5,2 \pm 2,0 \mathrm{ab}$ \\
\hline 9- Gen 99TG3450 & $0,00 \pm 0,0$ & $46,2 \pm 16,2 \mathrm{ab}$ & $26,0 \pm 8,2$ & $6,7 \pm 3,0 \mathrm{ab}$ \\
\hline 10- Gen 99TG883 & $5,25 \pm 5,3$ & $23,5 \pm 2,4 a b c$ & $5,7 \pm 3,4$ & $3,7 \pm 1,7 \mathrm{ab}$ \\
\hline 11- Gen 99TGR609 & $0,00 \pm 0,0$ & $17,7 \pm 3,3 \mathrm{bc}$ & $11,2 \pm 2,8$ & $10,5 \pm 3,9 \mathrm{ab}$ \\
\hline 12- IAC Jaraguá & $3,00 \pm 1,6$ & $68,2 \pm 5,9 \mathrm{a}$ & $18,7 \pm 18,0$ & $17,2 \pm 2,1 \mathrm{a}$ \\
\hline 13- Gen 95A10061531 & $0,75 \pm 0,8$ & $5,0 \pm 5,5 c$ & $8,7 \pm 4,6$ & $2,5 \pm 0,6 \mathrm{ab}$ \\
\hline 14- Gen TGR3114 & $15,20 \pm 14,9$ & $8,5 \pm 1,1 \mathrm{c}$ & $4,2 \pm 3,4$ & $3,2 \pm 1,3 \mathrm{ab}$ \\
\hline 15- Gen 96A1473153V2 & $0,00 \pm 0,0$ & $5,2 \pm 15,8 \mathrm{c}$ & $20,7 \pm 1,5$ & $4,2 \pm 2,0 \mathrm{ab}$ \\
\hline F (Tratamentos) & $0,91^{\mathrm{NS}}$ & $5,62^{* *}$ & $1,75^{\mathrm{NS}}$ & $2,09^{*}$ \\
\hline C. V. & 102,50 & 33,21 & 47,50 & 45,80 \\
\hline
\end{tabular}

1- Médias seguidas da mesma letra na coluna, não diferem entre si pelo teste de Tukey a $5 \%$ de probabilidade.

2- Para analise os dados foram transformados em $(x+0,50)^{1 / 2}$. 
Com relação ao número médio de ninfas de $C$. phaseoli em dez folíolos em quatro avaliações, observa-seque não ocorreu diferença entre os genótipos em nenhuma avaliação realizada (Tabela 3 ), mas no geral destaca-se a tendência de menor ocorrência do inseto nos genótipos IAC Harmonia e Gen 99TG2868 e maiores tendências de infestações nos genótipos IAC Jaraguá e Gen TGR3114.

Tabela 3 - Média do número de ninfas deCaliothrips phaseoli (Hood.) em dez folíolos, obtidos em 15 genótipos de feijoeiro, em quatro amostragens, Jaboticabal, SP, 2006.

\begin{tabular}{lcccc}
\hline Genótipo & \multicolumn{4}{c}{ Dias após a emergência das plantas ${ }^{1}$} \\
\cline { 2 - 5 } & 21 dias & 28 dias & 35 dias & 42 dias \\
\hline 1- IAPAR 31 & $9,25 \pm 5,6$ & $50,25 \pm 19,4$ & $42,00 \pm 20,2$ & $10,50 \pm 2,4$ \\
2- Rosinha G2 & $11,00 \pm 3,0$ & $53,50 \pm 5,4$ & $35,75 \pm 12,7$ & $14,50 \pm 4,3$ \\
3- Jalo precoce & $3,75 \pm 1,4$ & $48,50 \pm 8,2$ & $44,25 \pm 35,7$ & $9,50 \pm 2,8$ \\
4- Pérola & $19,25 \pm 7,4$ & $17,25 \pm 5,3$ & $40,25 \pm 10,6$ & $20,25 \pm 4,6$ \\
5- IAC Harmonia & $16,50 \pm 5,3$ & $32,75 \pm 13,5$ & $23,00 \pm 9,4$ & $17,25 \pm 1,5$ \\
6- Gen 99TGR110 & $6,25 \pm 1,8$ & $45,00 \pm 14,6$ & $53,25 \pm 16,9$ & $31,50 \pm 14,5$ \\
7- Gen 99TG2868 & $8,25 \pm 3,1$ & $19,75 \pm 3,2$ & $48,25 \pm 19,8$ & $14,50 \pm 2,9$ \\
8- Gen 99TGR3416 & $7,50 \pm 1,6$ & $50,75 \pm 17,6$ & $54,75 \pm 15,0$ & $23,25 \pm 6,2$ \\
9- Gen 99TG3450 & $10,25 \pm 1,9$ & $44,00 \pm 14,6$ & $38,00 \pm 22,1$ & $13,00 \pm 5,6$ \\
10- Gen 99TG883 & $21,75 \pm 13,8$ & $36,00 \pm 8,1$ & $43,00 \pm 15,3$ & $21,00 \pm 6,5$ \\
11- Gen 99TGR609 & $4,75 \pm 0,9$ & $41,75 \pm 16,0$ & $25,50 \pm 12,3$ & $21,25 \pm 4,4$ \\
12- IAC Jaraguá & $26,75 \pm 9,0$ & $55,00 \pm 14,8$ & $56,75 \pm 15,1$ & $17,25 \pm 5,8$ \\
13- Gen 95A10061531 & $19,25 \pm 3,1$ & $63,00 \pm 11,8$ & $31,25 \pm 11,2$ & $29,50 \pm 13,3$ \\
14- Gen TGR3114 & $27,25 \pm 8,3$ & $36,75 \pm 18,0$ & $43,75 \pm 10,1$ & $34,00 \pm 9,1$ \\
15- Gen 96A1473153V2 & $29,75 \pm 9,0$ & $53,50 \pm 15,7$ & $24,25 \pm 7,7$ & $14,75 \pm 1,8$ \\
\hline F (Tratamentos) & $2,58^{\mathrm{NS}}$ & 1,14 Ns & 0,63 Ns & 1,21 Ns \\
C. V. & 38,14 & 31,06 & 38,11 & 33,12
\end{tabular}

1- Para análise os dados foram transformados em $(x+0,50)^{1 / 2}$.

Tabela 4 - Média do número de ovos e ninfas de B. tabaci (Genn.) biótipo b e de ninfas de Caliothrips phaseoli (Hood.) em dez folíolos, obtidos em 15 genótipos de feijoeiro, na média de quatro amostragens, dos 21 aos 42 DAE, Jaboticabal, SP, 2006.

\begin{tabular}{lccc}
\hline Genótipo & Ovos de B. tabaci biótipo B & Ninfas de B. tabaci biótipo B & Ninfas de C. Phaseoli \\
\hline 1- IAPAR 31 & $31,6 \pm 11,6 \mathrm{abc}$ & $16,7 \pm 7,2 \mathrm{ab}$ & $28,0 \pm 10,6$ \\
2- Rosinha G2 & $11,4 \pm 6,0 \mathrm{~cd}$ & $5,7 \pm 2,9 \mathrm{ab}$ & $28,7 \pm 9,9$ \\
3- Jalo precoce & $16,8 \pm 5,8 \mathrm{bcd}$ & $4,9 \pm 1,9 \mathrm{ab}$ & $26,5 \pm 11,6$ \\
4- Pérola & $9,6 \pm 4,9 \mathrm{~d}$ & $4,2 \pm 1,6 \mathrm{~b}$ & $24,1 \pm 5,4$ \\
5- IAC Harmonia & $7,1 \pm 3,9 \mathrm{~d}$ & $4,6 \pm 3,1 \mathrm{~b}$ & $22,4 \pm 3,8$ \\
6- Gen 99TGR110 & $12,8 \pm 3,4 \mathrm{~cd}$ & $11,9 \pm 6,2 \mathrm{ab}$ & $34,0 \pm 10,3$ \\
7- Gen 99TG2868 & $14,3 \pm 5,5 \mathrm{~cd}$ & $9,8 \pm 4,8 \mathrm{ab}$ & $22,7 \pm 8,8$ \\
8- Gen 99TGR3416 & $20,6 \pm 6,6 \mathrm{abcd}$ & $13,6 \pm 7,0 \mathrm{ab}$ & $34,1 \pm 11,3$ \\
9- Gen 99TG3450 & $38,4 \pm 9,6 \mathrm{ab}$ & $19,8 \pm 10,4 \mathrm{ab}$ & $26,3 \pm 8,6$ \\
10- Gen 99TG883 & $14,9 \pm 8,8 \mathrm{~cd}$ & $9,6 \pm 4,7 \mathrm{ab}$ & $30,4 \pm 5,4$ \\
11- Gen 99TGR609 & $16,5 \pm 8,0 \mathrm{~cd}$ & $9,9 \pm 3,7 \mathrm{ab}$ & $23,3 \pm 7,6$ \\
12- IAC Jaraguá & $44,5 \pm 13,8 \mathrm{a}$ & $26,8 \pm 14,3 \mathrm{a}$ & $38,9 \pm 10,0$ \\
13- Gen 95A10061531 & $9,0 \pm 6,3 \mathrm{~d}$ & $5,4 \pm 2,2 \mathrm{ab}$ & $35,8 \pm 9,5$ \\
14- Gen TGR3114 & $9,4 \pm 4,8 \mathrm{~d}$ & $7,8 \pm 2,7 \mathrm{ab}$ & $38,6 \pm 4,9$ \\
15- Gen 96A1473153V2 & $17,0 \pm 6,8 \mathrm{bcd}$ & $7,6 \pm 4,5 \mathrm{ab}$ & $30,9 \pm 8,2$ \\
\hline F (Tratamentos) & $6,88^{* *}$ & $2,21^{*}$ & $1,27^{\mathrm{N}}$ \\
C. V. & 23,28 & 35,03 & 18,04 \\
\hline
\end{tabular}

1- Médias seguidas da mesma letra na coluna não diferem entre si pelo teste de Tukey a 5\% de probabilidade.

2- Para análise os dados foram transformados em $(x+0,50)^{1 / 2}$ 
Esses dados são semelhantes aos de BoIçA JúnIOR et al . (2005)emqueessesautoresavaliaramainteraçãodeóleos vegetais com inseticida no controle de Thripstabaci (Lind.) no genótipo Carioca e encontraram valores baixos de ninfas eadultos da praga nes sa mesma época decultivo.

Analisando-se os valores médios das avaliações realizadas para o número de ovos e ninfas de B. tabaci biótipo B e ninfas de C. phaseoli, independente da data de amostragem (Tabela 4), verificam-se diferenças para o primeiro esegundo parâmetro(ovos eninfas de mosca branca). Com relação ao primeiro, observa-se que o genótipo IAC Jaraguá foi o mais preferido pela mosca-branca para oviposição, com 44,5 ovos em média, enquanto que os genótipos IAC Harmonia, Gen 95A10061531, Gen TGR3114 e Pérola foram os menos preferidos para oviposição da praga, $\operatorname{com} 7,1$; 9,0; 9,4 e 9,6 ninfas em média, respectivamente, nas quatro avaliações realizadas.

Em relação ao número médio de ninfas de moscabranca nos genótipos estudados, observa-se diferença significativa entre os tratamentos, sendo o genótipo IAC Jaraguá o mais infestado pela praga, com 26,8 ninfas de média, e os genótipos Pérola e IAC Harmonia os de menores infestações, com 4,2 e 4,6 ninfas em média, respectivamente.

Para o número médio da infestação de ninfas de $C$. phaseoli observa-se que não ocorreu diferença, porém destaca-se uma maior tendência de infestação no genótipo IAC Jaraguá, com 38,9 ninfas, já o genótipo IAC Harmonia comportou-se de forma contrária, apresentando tendência de menor infestação, com índice de 22,4 ninfas em média.

Pela análise de regressãolinear entre a temperatura média (Tm) e o número de ninfas de $B$. tabaci biótipo $B$ $(\mathrm{Nn})$ e ninfas de $C$. phaseoli $(\mathrm{Nt})$, verificam-se correlações lineares negativas não significantes entre Tm versus $\mathrm{Nn}\left(\mathrm{y}=4,6764+0,64 \mathrm{x}, \mathrm{R}=-0,118^{\mathrm{ns}}\right)$ e Tm versus $\mathrm{Nt}\left(\mathrm{y}=4,8228+0,023 \mathrm{x}, \mathrm{R}=-0,344^{\mathrm{ns}}\right)$, indicando que a temperatura média não acarreta diminuição na infestação das pragas estudadas. TOMASO (1993), estudando o potencial de infestação de B. tabaci biótipo Bno feijoeiro em função de plantas hospedeiras econdições climáticas, encontrou dados diferentes a esses apresentados, mostrando quea variável climática temperatura influencia na flutuação populacional da praga.

\section{CONCLUSÕES}

- Os genótipos menos ovipositados pela B. tabaci biótipo B foram IAC Harmonia, Pérola, Gen TG3114 e Gen 95A10061531, enquanto os mais ovipositados foram IAC Jaraguá e Gen 99TG3450.

- As menores infestações de ninfas de B. tabaci biótipo B foram observadas em Pérola e IAC Harmonia e a maior em IAC Jaraguá.
- Não foi verificada correlação linear significativa entre temperatura média semanal e número total de ninfas de B. tabaci biótipo B e C. phaseoli.

\section{AGRADECIMENTOS}

À pesquisadora Renata Chiarini Monteiro Cônsoli, pela identificação da espécie Caliothrips phaseoli.

\section{REFERÊNCIAS}

BOIÇA JÚNIOR, A.L.; VENDRAMIM, J.D. Desenvolvimento de Bemisia tabaci em genótipos de feijão. Anais da Sociedade Entomológica do Brasil, v.15, p.231238, 1986.

BOIÇA JÚNIOR, A.L.; SANTOS, T.M.; MUÇOUÇAH, M.J. Adubação e inseticidas no controle de Empoasca kraemeri Ross \& Moore, 1957 e Bemisia tabaci (Gennadius, 1889), em cultivares de feijoeiro semeado no inverno. Sciencia Agrícola, v.57, p.635-641, 2000.

BOIÇA JÚNIOR, A.L.; ANGELINI, M.R.; COSTA, G.M.; BARBOSA, J.C. Efeito do uso de óleos vegetais, associados ou não a inseticida, no controle de Bemisia tabaci (Genn.) e Thrips tabaci (Lind.), em feijoeiro, na época "das secas". Boletin del Sanidad Vegetal Plagas, v.31, p.449-458, 2005.

CAMPOS, Z.R.; BOIÇA JÚNIOR, A.L.; LOURENÇÃO, A.L.; CAMPOS, A.R. Fatores que afetam a oviposição de Bemisia tabaci (Genn.) biótipo B (Hemiptera: Aleyrodidae) em algodoeiro. Neotropical Entomology, v.34, p.823-827, 2005.

CZEPAK, C.; FERNANDES, P.M.; SILVEIRA, C.A. Eficiência dos inseticidas Gaucho 600 SC, Gaúcho 700 PM e Provado 200 SC no controle de Bemisia argentifolli na cultura do feijão. In REUNIÃO NACIONAL DE PESQUISA DE FEIJÃO, 6., 1999, Goiânia. Resumos. Goiânia: Embrapa Arroz e Feijão, 1999. p.118-119.

DITTRICH, V.; ERNST, G.H.; RUESCH, O.; UK, S. Resistance mechanisms in sweetpotato whitefly (Homoptera: Aleyrodidae) populations from Sudan, Turkey, Guatemala, and Nicaragua. Journal of Economic Entomology, v.83, p.665-1670, 1990.

FARIA, J.C.; ANJOS, J.R. N.; COSTA, A.F.; SPERÂNCIO, C.A.; COSTA, C.L. Doenças causadas por vírus e seu controle. In: ARAUJO, R.S.; RAVA, C.A.; STONE, DROST, YC.; VAN LENTEREN, J.C.; VAN ROERMUND, H.J.W. Life history parameters of different biotypes of Bemisia tabaci (Hemiptera: Aleyrodidae) in relation to temperature and host plant: a selective review. Bulletin of Entomological Research, v. 88, p.219-229, 1998. 
FARIA, J.C.; ZIMMERMANN, M.J.O. Controle do mosaico dourado do feijoeiro (Phaseolus vulgaris) pela resistência varietal e inseticidas. Fitopatologia Brasileira, v.13, p.32-35, 1987.

FARIA, J.C.; OLIVEIRA, M.N.; YOKOYAMA, M. Resposta comparativa de genótipos de feijoeiro (Phaseolus vulgaris) a inoculação com o vírus do mosaico dourado no estágio de plântulas. Fitopatologia Brasileira, v.19, p.566-572, 1994.

GALLO, D; NAKANO, O.; SILVEIRA NETO, S.; BAPTISTA, G.C.; BERTI FILHO, E.; PARRA, J.R.P.; ZUCCHI, R.A.; ALVES, S.B.; VENDRAMIM, J.D.; MARCHINI, L.C.; LOPES, J.R.S.; OMOTO, S. Entomologia agrícola. Piracicaba: FEALQ, 2002. 920p.

JESUS, F.G.; BOIÇA JÚNIOR, A.L.; STEIN, C.P.; CARBONELL, S.A.M., ANGELLINI, M.R.; CHAGAS FILHO, N.R; MORENO, D.B. Avaliação da infestação de Bemisia tabaci (Genn.) biótipo B (Hemiptera: Aleyrodidae) em genótipos de feijoeiro, em condições de campo. In: SIMPÓSIO DE PLANTAS DANINHAS, DOENÇAS E PRAGAS DA CULTURA DO FEIJOEIRO, 2006, Campinas. Anais. 2006. CD-ROM.

LARA, F.M. Princípios de resistência de plantas a insetos. São Paulo: Ícone, 1991. 336p.

LEMOS, L.B.; FORNASIERI FILHO, D.; SILVA, T.R.B.; SORATO, R.P. Suscetibilidade de genótipos de feijão ao vírus do mosaico dourado. Pesquisa Agropecuária Brasileira, v.38, p.575-581, 2003.

MAGALHÃES, B.P.; CARVALHO, S.M. Insetos associados à cultura. In: ZIMMERMANN, M.J. DE O., ROCHA, M., YAMADA, T. (Ed.). Cultura do feijoeiro: fatores que afetam a produtividade. Piracicaba: Associação Brasileira para pesquisa da Potassa e do Fosfato, 1998. p.573589.

MCAUSLANE, H.J. Influence of leaf pubescence on ovipositional preference of Bemisia argentifolii (Homoptera: Aleyrodidae) on soybean. Environmental Entomology, v.25, p.834-841, 1996.
PRABHAKER, N.; COUDRIET, D.L.; MEYERDIRK, D.E. Insecticide resistance in the sweetpotato whitefly, Bemisia tabaci (Homoptera: Aleyrodidae). Journal of Economic Entomology, v.78, p.748-752, 1985.

PRABHAKER, N.; TOSCANO, N.C.; COUDRIET, D.L. Susceptibility of the immature and adult stages of the sweet potato whitefly (Homoptera: Aleyrodidae) to selected insecticides. Journal of Economic Entomology, v.82, p.983-988, 1989.

SALGUERO, V. Perspectivas para el manejo del complejo mosca blanca - virosis. In: HILJE, L.; ARBOLEDA, O. Las moscas blancas (Homoptera: Aleyrodidae) em America Central y Caribe. Turrialba: CATIE, 1993. p.20-26. (Informe Técnico, 205).

TOSCANO, L.C.; BOIÇA JÚNIOR, A.L.; MARUYAMA, W.I. Fatores que afetam a oviposição de Bemisia tabaci (Genn.) biótipo B (Hemiptera: Aleyrodidae) em tomateiro. Neotropical Entomology, v.31, p.631-634, 2002.

TOMASSO, C.A. Potencial de infestação de Bemisia tabaci (Genn. 1889) (Hemiptera: Aleyrodidae) no feijoeiro $m$ função e plantas hospedeiras e nas condições climáticas, na região de Jaboticabal, SP. 1993. 83f. Monografia (Graduação em Agronomia) - Faculdade de Ciências Agrárias e Veterinárias de Jaboticabal, Universidade Estadual Paulista, Jaboticabal, 1993.

VIEIRA, C.; PAULA JÚNIOR, T.J.; BORÉM, A. Feijão: aspectos gerais e cultura no Estado de Minas. Viçosa: UFV, 1998. 596p.

WALKER, G.P.; PERRING, T.M. Feeding and oviposition behavior of whiteflies (Homoptera:Aleyrodidae) interpetred from AC electronic feeding monitor waveforms. Annals of the Entomological Society of America, v.18, p.363-374, 1994.

YOKOYAMA, M. Pragas. In: VIEIRA, C.; PAULA JÚNIOR, T.J.; BORÉM, A. (Ed.). Feijão. 2.ed. Viçosa: UFV, 2006. p.341-357.

Recebido em 28/1/08

Aceito em 16/5/09 